\title{
Ultrasound-modulated optical tomography for dense turbid media
}

\section{Lihong V. Wang, Xuemei Zhao, Steven L. Jacques}

Lihong V. Wang, Xuemei Zhao, Steven L. Jacques, "Ultrasound-modulated optical tomography for dense turbid media," Proc. SPIE 2676, Biomedical Sensing, Imaging, and Tracking Technologies I, (24 April 1996); doi: 10.1117/12.238787

SPIE. Event: Photonics West '96, 1996, San Jose, CA, United States 


\title{
Ultrasound-Modulated Optical Tomography of Dense Turbid Media
}

\author{
Lihong Wang, Ph. D. ${ }^{1}$ \\ Xuemei Zhao, M. S. ${ }^{2}$ \\ Steven L. Jacques, Ph. D. ${ }^{2}$
}
(1) Bioengineering Program
234C Zachry Engineering Center
Texas A\&M University, College Station, Texas 77843, USA
Email: lwang@aggie.tamu.edu
(2) Laser Biology Research Laboratory
University of Texas M. D. Anderson Cancer Center
1515 Holcombe Boulevard, Houston, Texas 77030, USA

\begin{abstract}
Continuous-wave ultrasonic modulation of scattered laser light has been used to image objects in tissue-simulating turbid media for the first time. ${ }^{1}$ We hypothesized that the ultrasound wave focused into the turbid media modulates the laser light passing through the ultrasonic focal zone. The modulated laser light collected by a photomultiplier tube reflects the local mechanical and optical properties in the focal zone. Buried objects in 5-cm thick tissue phantoms (absorption coefficient $\mu_{\mathrm{a}}=0.1 \mathrm{~cm}^{-1}$, reduced scattering coefficient $\mu_{\mathrm{s}}{ }^{\prime}=10 \mathrm{~cm}^{-1}$ ) were located with millimeter resolution by scanning and detecting alterations of the ultrasound-modulated optical signal.
\end{abstract}

\section{Key Words}

Ultrasound-modulation, optical imaging, optical tomography, turbid media, scattering media, biological tissue phantoms. 


\section{Introduction}

\section{Non-Optical Imaging}

Breast cancer is the most common malignant neoplasm and the leading cause of cancer deaths in women in the United States. A means for prevention of breast cancer has not been found, and early detection and treatment is the best solution to improve cure rate. At present, mammography and ultrasonography are clinically used for breast cancer detection. Mammography is currently the only reliable means of detecting nonpalpable breast cancers. As a supplementary tool, ultrasound is used to evaluate the internal matrix of circumscribed masses found at mammography or of palpable masses that are obscured by radiographically dense parenchyma at mammography. ${ }^{2-6}$ However, $\mathrm{x}$-ray mammography is ionizing radiation, and imaging of radiographically dense breasts is difficult. Ultrasonography cannot detect many of the nonpalpable cancers that are not visible on mammograms of good quality. ${ }^{3}$

Several other techniques are under investigation for breast cancer imaging. Magnetic resonance imaging (MRI) offers great promise for imaging of the radiographically dense breast. $^{7,8}$ Breast MRI is superior to mammography in differentiating solid from cystic lesions and is equivalent to mammography in providing information regarding different parenchymal patterns. Injection of intravenous contrast material with MRI increases cancer detectability in spite of the fact that breast cancer and glandular tissues have similar magnetic resonance tissue characteristics. However, breast MRI is expensive, has inferior spatial resolution to mammography, and cannot image microcalcifications, which are often the sole indicators of breast cancer., 3

Breast computed tomography (CT) has been investigated for the differentiation of benign from malignant solid masses. Breast CT involves the use of intravenous injection of iodinated contrast material and has limited spatial resolution and high cost; hence, it is not suited for routine breast cancer screening. ${ }^{3}$

\section{Optical Imaging}

Nonionizing laser light detection of breast cancer is a new and active field. ${ }^{10-34}$ The optical properties of normal and diseased breast tissues are usually different; ${ }^{35,36}$ therefore, it is possible to detect breast cancers based on the measurement of optical properties. For example, the scattering coefficient of fibrocystic tissue $\left(\sim 600 \mathrm{~cm}^{-1}\right)$ is approximated $50 \%$ higher than that of normal glandular breast tissue $\left(\sim 400 \mathrm{~cm}^{-1}\right)$ or $100 \%$ higher than that of normal breast adipose tissue $(\sim 300$ $\left.\mathrm{cm}^{-1}\right)$ in the wavelength range of $500-1100 \mathrm{~nm}$; the absorption coefficient of ductal carcinoma $(\sim 4$ $\left.\mathrm{cm}^{-1}\right)$ is about $100 \%$ higher than the coefficients of normal glandular or adipose breast tissues $(\sim 2$ $\mathrm{cm}^{-1}$ ) around $550 \mathrm{~nm}$ wavelength. The optical difference is not surprising because cancerous tissues manifest significant architectural changes at the cellular and sub-cellular levels, and the cellular components that cause elastic scattering have dimensions typically on the order of visible to near-IR wavelengths. Some tumors are associated with vascularization, where blood causes increased light absorption. The use of optical contrast agents can also be exploited to enhance the optical contrast between normal and abnormal tissues. ${ }^{14}$

Because tissues are optically turbid media, light is quickly diffused inside tissues as a result of scattering. Light transmitted through tissues is classified into three categories: ballistic light, quasi-ballistic light, and diffuse light. Ballistic light experiences no scattering by tissue and thus travels straight through the tissue. Quasi-ballistic light carries some imaging information. Diffuse light carries little direct imaging information and overshadows ballistic or quasi-ballistic light.

One of the techniques is called "early-photon imaging". $2,11,15,24$ If diffuse light is rejected, and ballistic or quasi-ballistic light is collected, buried objects can be detected. This technique uses a short-pulse laser $(<1 \mathrm{ps}$ pulse width) to illuminate the breast tissue. Only the initial portion of transmitted light is allowed to pass to a light detector, and the late-arriving light is gated off by a fast optical gate. If only ballistic light is detected, the imaging is called ballistic imaging. It has been shown that ballistic imaging is possible only for breast tissue thickness less than $0.14 \mathrm{~cm}$ or 42 mean free paths (mfp). ${ }^{7,39}$ Most ballistic imaging techniques reported in the literature have 
achieved approximately $30 \mathrm{mfp} .^{18,22,24}$ Therefore, this approach is suitable for thin tissue samples but suffers loss of signal and resolution for thick tissues as a result of the strong scattering of light by the tissue. A straightforward continuous-wave laser imaging technique has also achieved 30 $\mathrm{mfp}^{38}$

For breast tissue of practical thickness $(5$ to $10 \mathrm{~cm}$ ), scattered light must be used to image breast cancers. We have demonstrated that for a 5-cm-thick breast tissue, the detector must collect light that has experienced at least 1100 scattering events in tissue to yield enough signal. ${ }^{37}$ However, if a 1-mW visible or near IR laser is incident on one side of a 5-cm thick breast tissue (absorption coefficient $\mu_{\mathrm{a}}=0.1 \mathrm{~cm}^{-1}$, reduced scattering coefficient $\mu_{\mathrm{s}}{ }^{\prime}=10 \mathrm{~cm}^{-1}$ ), we have estimated using diffusion theory that the diffuse transmittance on the other side is on the order of 1 $\mathrm{nW} / \mathrm{cm}^{2}$ or $10^{9}$ photons $/\left(\mathrm{s} \cdot \mathrm{cm}^{2}\right)$, which is easy to detect using a PMT capable of single-photon counting.

Imaging resolution of pure laser imaging degrades linearly with increased tissue thickness. ${ }^{39}$ Hebden et al detected the temporal profiles of the scattered light using a streak camera and then mathematically extrapolated the signal to the early part of the profiles in which the actual light could not be detected. ${ }^{13}$ The early portion of the profiles were integrated to construct the images of buried objects (8-mm diameter) in a turbid medium (51-mm thick). The spatial resolution was found to be $5 \mathrm{~mm}$ for a $5-\mathrm{cm}$ thick breast phantom, ${ }^{40}$ which agrees with the theoretical prediction. ${ }^{39}$ This time-domain technique requires expensive short-pulse lasers and fast light detectors.

Another technique for laser light imaging is the frequency-domain technique, also called photon-density wave imaging, which was first introduced into the field by Gratton et al..$^{10,19,30-34}$ This technique uses amplitude-modulated laser light (at approximately $100 \mathrm{MHz}$ ) to illuminate the tissue and detect the diffuse light. Chance and Yodh et al have recently reconstructed the images of the buried objects based on this frequency-domain technique. ${ }^{41}$ This technique has similar imaging resolution as the time-domain technique but requires complex imaging reconstruction algorithms.

Marks et al have investigated tissue imaging using the combination of pulsed ultrasound and laser light. ${ }^{42}$ They detected the signal of a homogeneous turbid medium without buried objects. We developed a new approach: ultrasound-modulated optical tomography that combines continuous-wave rather than pulsed-ultrasound and laser irradiation. We have successfully imaged buried objects in tissue-simulating turbid media. ${ }^{1}$ The major advantage of using continuous-wave ultrasound modulation over pulsed ultrasound modulation is the significant increase in signal-tonoise ratio, which allows us to image buried objects in turbid media

Ultrasound-modulated optical tomography measures mechanical and optical properties of tissues, whereas $\mathrm{x}$-ray mammography measures radiographical density of tissues and ultrasonography measures echogenicity of tissues. If the tumors are mechanically or optically different than the surrounding normal tissues, this method should be able to image the tumors. Studies have shown the mechanical and optical differences between many normal and abnormal tissues. ${ }^{43-45 ; 35,36}$ Our technique is, therefore, an addition to the current techniques for breast imaging. Furthermore, this approach is free of the hazards of ionizing radiation with mammography. Ultrasound-modulated optical tomography separates the resolution and signal problem of light-only imaging because the resolution is determined by the size of the ultrasound focal spot and the signal depends on any light passing through the ultrasound focal spot (including ballistic, quasi-ballistic light, and diffuse light). If the clinical trial succeeds, this technique will provide a good tool for breast cancer screening, detection, monitoring, and therapy planning.

In summary, the advantages of ultrasound-modulated optical tomography over other purely optical imaging methods are (Table 1): (1) good resolution: capability to image 5-cm thick breast phantoms with $\sim 1$-mm resolution and $\sim 65 \%$ contrast with the potential for improvement; (2) costeffectiveness; (3) detection of combined mechanical and optical properties; and (4) no need for complex inverse algorithms. The advantages of ultrasound-modulated optical tomography over xray mammography are: (1) non-ionization radiation; (2) low-cost; and (3) capability to detect optical spectral information of tumors, which is related to tumor physiology. The imaging resolution of different medical diagnostic tools is listed in Fig. 1. 
Table 1. Comparison of different optical imaging methods.

\begin{tabular}{l|l|l|l}
\hline Method & Imaging Resolution & Cost & Comments \\
\hline \hline Time-gated & $\sim 5 \mathrm{~mm}$ for 5-cm thick tissue & High & Fast laser and detector \\
\hline Frequency-domain & $\sim 5 \mathrm{~mm}$ for 5-cm thick tissue & Medium & Complex algorithm \\
\hline Ultrasound-modulated & $\sim 1 \mathrm{~mm}$ for 5-cm thick tissue & Low & Simple algorithm \\
\hline
\end{tabular}

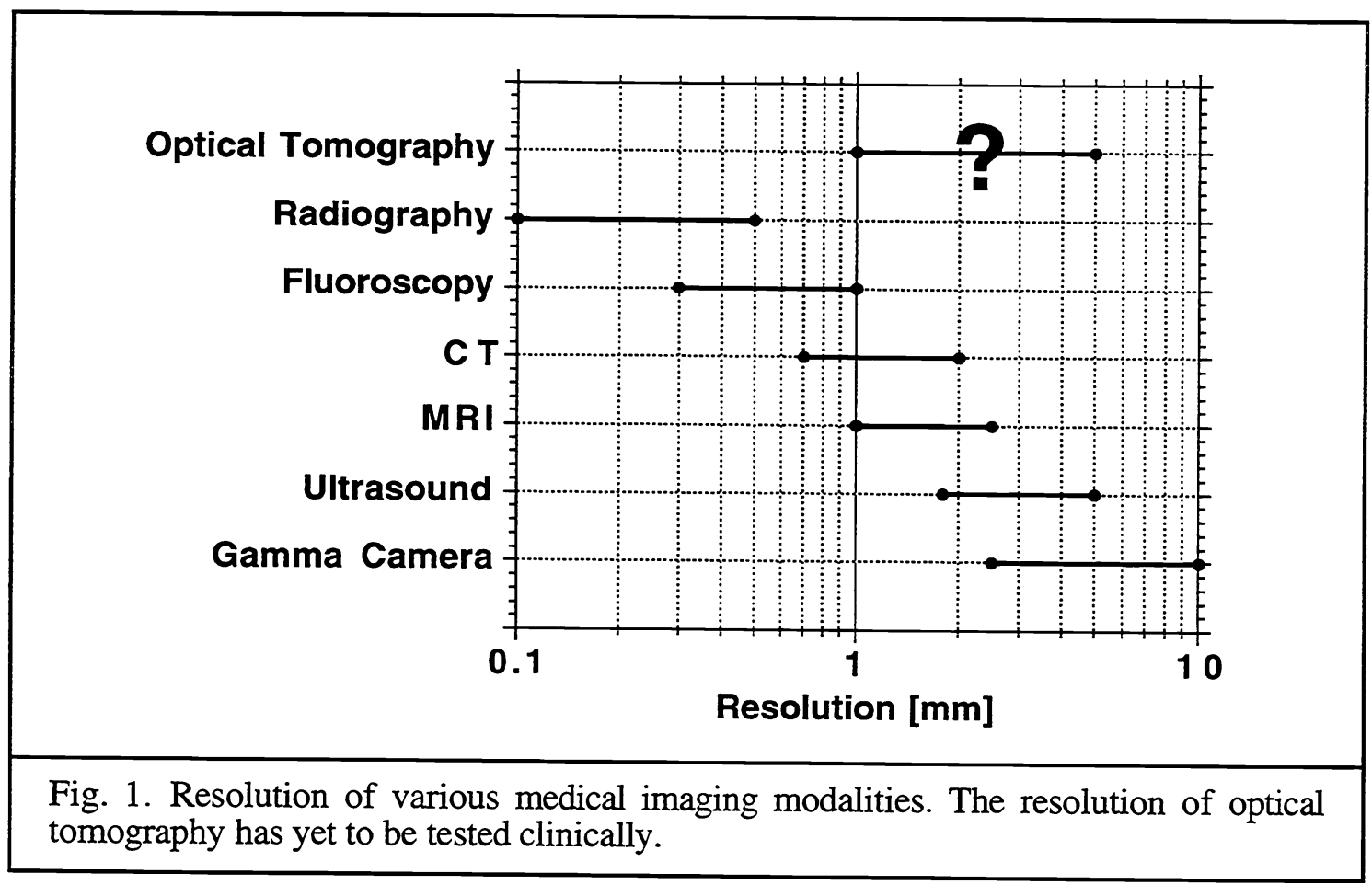

The underlying physics of ultrasound-modulated optical tomography is acousto-optics in turbid media. Although acousto-optics in clear media has been well studied ${ }^{46,47}$ turbid acoustooptics is a new field, which may find applications in medicine, underwater detection, atmosphere optics, and other fields involving turbid media.

\section{Methods and Materials}

\section{Hypothesis}

The design of the experiment on ultrasound-modulated optical tomography was based on the following hypothesis (Fig. 2). Continuous-wave ultrasound wave was propagated through a turbid medium and focused to a small spot inside the medium. The ultrasound wave modulated light passing through the ultrasonic field. Several possible modulation mechanisms are discussed below.

Ultrasound wave generated pressure variation. The pressure variation induced a density change in the medium as a result of the compressibility of the medium. The optical absorption and scattering coefficients were proportional to the number density of absorbers and scatterers, respectively. The index of refraction varied with the density as well. Therefore, the density variation modulated the optical properties of the solution at the ultrasound frequency. The variation of optical properties modulated the light passing through the ultrasonic field. 
Ultrasound wave generated particle displacement. The particle displacement caused optical pathlength to change. Coherent laser light passing through turbid media could generate speckles. Because speckles depended on the optical pathlength, the speckles varied at the ultrasonic frequency.

Ultrasound wave can be considered as phonons. The photon-phonon interaction caused Doppler shift of the optical frequency by the ultrasonic frequency. An optical detector functioned as a heterodyning device between the Doppler-shifted light and unshifted light and produced a signal of the ultrasonic frequency.

The modulated light carried the information of the optical and mechanical properties near the focal spot, where the modulation off focus was assumed less than that at the focus. The ultrasound-modulated light signal can be easily separated from the unmodulated light signal by an electronic filter. Scanning the imaging system relative to the turbid medium would generate an image of the medium based on the distribution of optical and mechanical properties. The ultrasound modulation depended on any light, which was primarily diffuse light rather than ballistic or quasiballistic light for dense turbid medium. The imaging resolution depended on the size of the ultrasonic focus.

The above modulation sequences are summarized in Fig. 3, where $p(t)$ is the ultrasonic pressure as a function of time, $N(t)$ the number density, $\mu_{\mathrm{a}}(\mathrm{t})$ the absorption coefficient, $\mu_{\mathrm{s}}{ }^{\prime}(\mathrm{t})$ the reduced scattering coefficient, $n(t)$ the index of refraction, and $I(t)$ the light intensity.

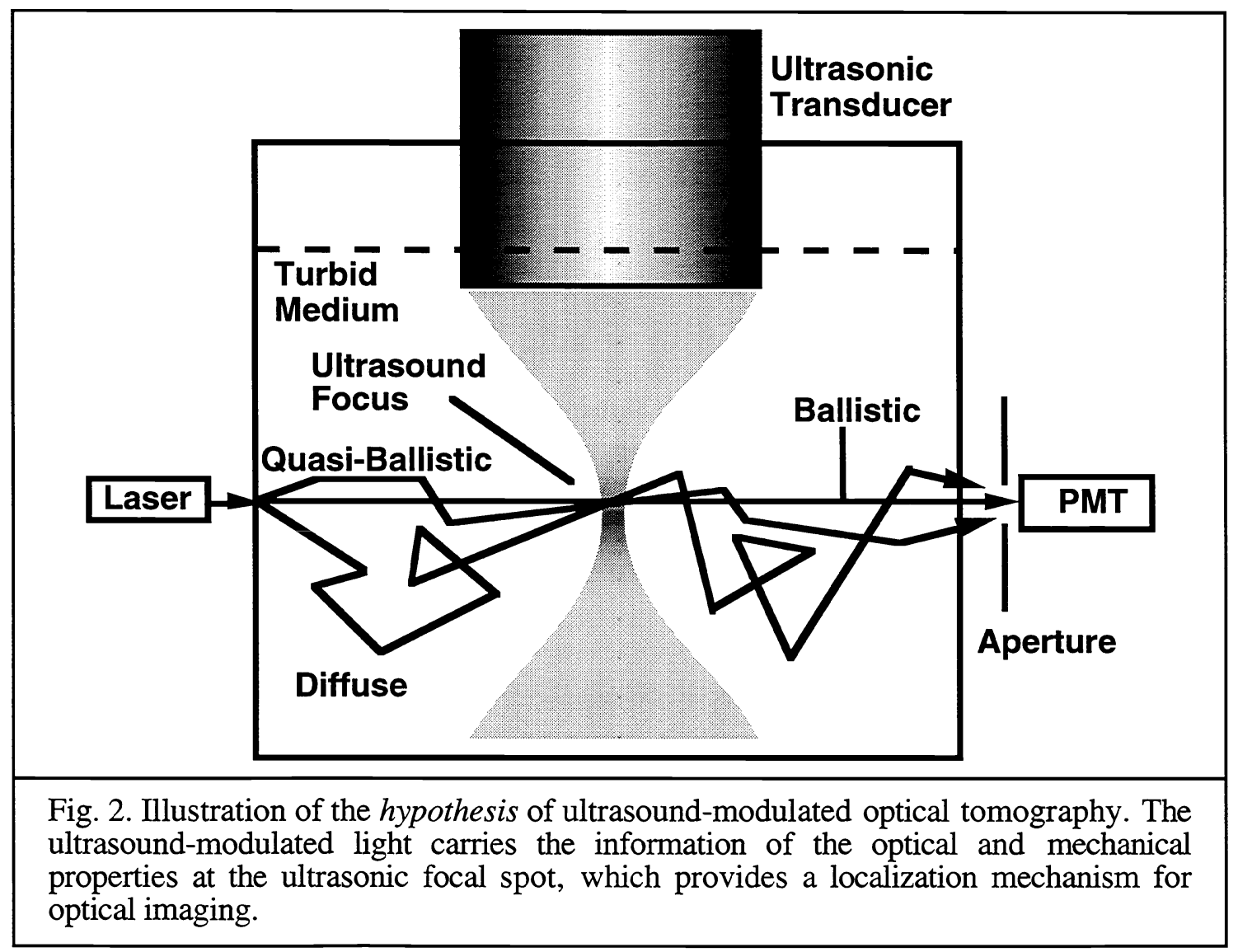




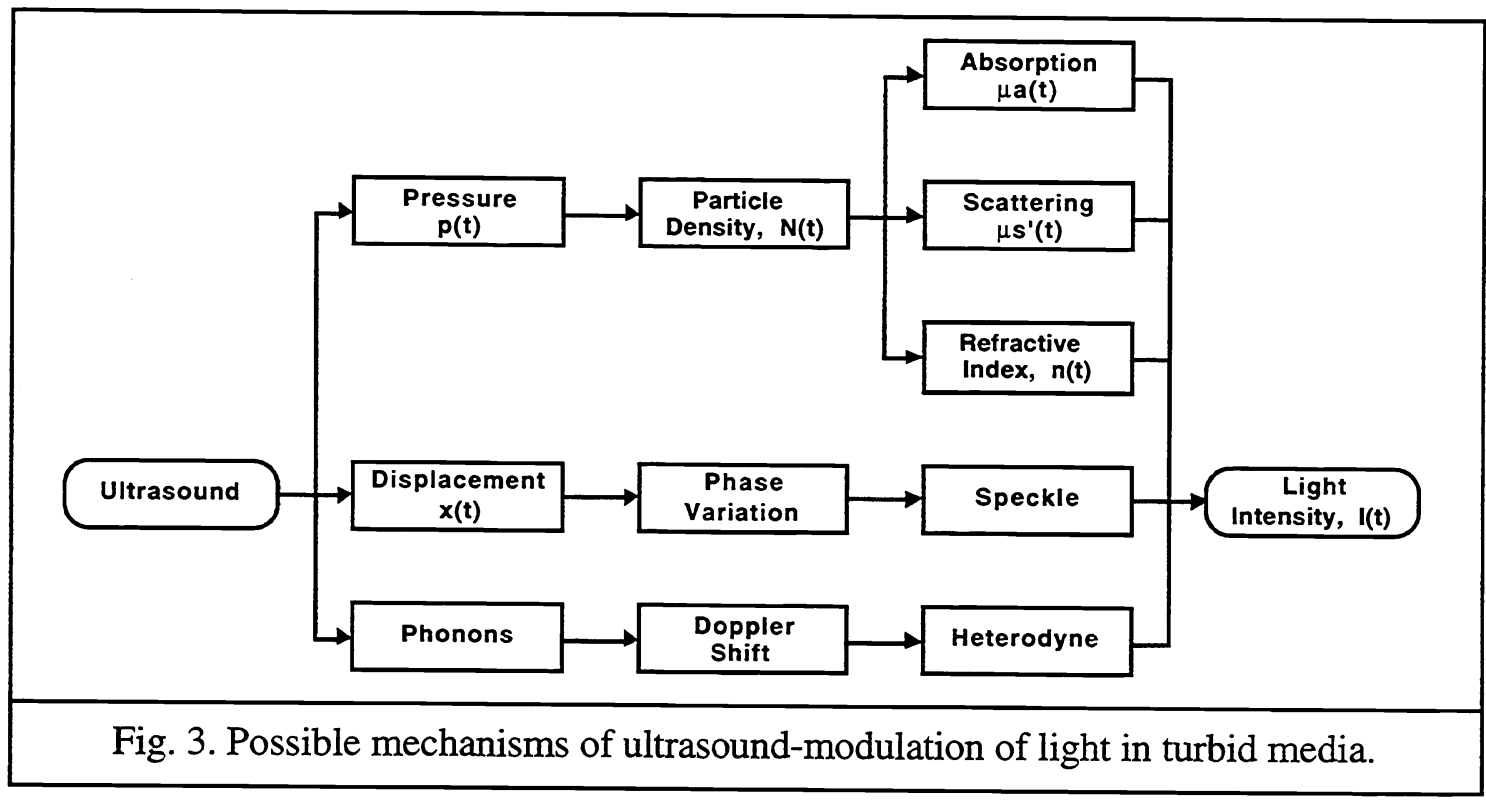

\section{Phantom Preparation}

Tissue phantoms (turbid media) were prepared to simulate the optical properties of tissues by dissolving dominantly absorbing Trypan blue dye and dominantly scattering Intralipid in water or $5 \%$ gel solution (by weight) for liquid and solid phantoms, respectively. The phantom was contained in a cuvette, which was $5 \mathrm{~cm}$ thick, $23 \mathrm{~cm}$ wide, and $18 \mathrm{~cm}$ high. Two ultrasonic absorbers ( $3.5 \mathrm{~cm}$ thick) were placed inside the two sides of the cuvette. Another ultrasonic absorber $(4.5 \mathrm{~cm}$ thick) was placed at the bottom of the cuvette. The gel phantom $(8 \mathrm{~cm}$ high) was placed inside the cuvette before it coagulated in a refrigerator. Another gel phantom cube $(7 \mathrm{~mm}$ each side) was placed inside the background gel in the mid-plane to simulate a buried object. The facets of the cube were parallel with those of the cuvette. A liquid phantom ( $3 \mathrm{~cm} \mathrm{high})$ that had identical optical properties as the background gel was poured into the cuvette to couple ultrasonic wave and allow the scanning of ultrasonic transducers.

The optical properties of the background gel and liquid phantoms were: $\mu_{\mathrm{a}}=0.1 \mathrm{~cm}^{-1}, \mu_{\mathrm{s}}{ }^{\prime}$ $=10 \mathrm{~cm}^{-1}$. The optical properties of the buried gel object phantom were: $\mu_{\mathrm{a}}=1.5 \mathrm{~cm}^{-1}, \mu_{\mathrm{s}}{ }^{\prime}=10$ $\mathrm{cm}^{-1}$. The background optical properties were close to the realistic optical properties of breast tissues. ${ }^{36}$

\section{Experimental Setup}

The experimental setup is shown in Fig. 4. The glass cuvette was seated on a twodimensional translation stage, which was able to scan the cuvette along both the $\mathrm{x}$ and $\mathrm{z}$ axes. While the cuvette was scanned, the rest of the system, including the optical and ultrasonic systems, were fixed.

The He-Ne laser (Uniphase, 1135P) with 10-mW output power and 632.8-nm wavelength delivered a single-mode Gaussian beam perpendicular to the front surface of the cuvette. The lower end of the ultrasonic transducer was buried in the solution to allow a good coupling of the ultrasound wave. The room lights were turned off to reduce the ambient noise collected by the photomultiplier tube (PMT, Hamamatsu, R928). The voltage supply of the PMT (Hamamatsu, HC 123-01) was powered with a 12-volt DC power supply, and the voltage on the PMT cathode was set to -850 volts. A $1-\mathrm{cm}$ aperture was placed in front of the PMT to limit light entering the PMT. 


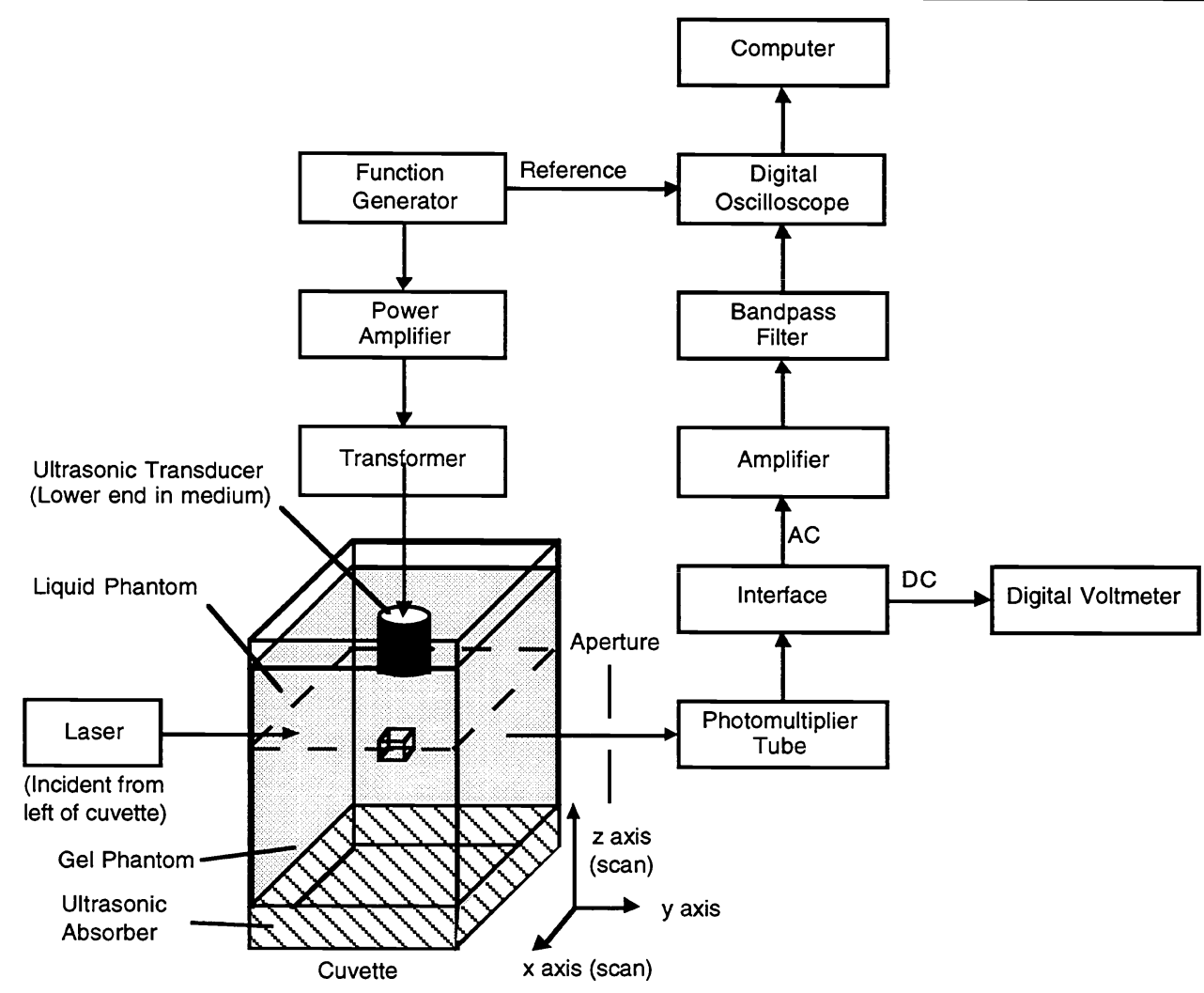

Fig. 4. Block diagram of the setup of ultrasound-modulated optical tomography. The arrows between blocks represent electrical signals, and the arrows from the laser and from the cuvette represent optical signals. Note the Cartesian coordinate system that will be used subsequently.

The function generator (Stanford Research Systems, DS345) produced a sinusoidal wave at a fixed frequency of $999,825 \mathrm{~Hz}$, which was the center frequency of the bandpass filter in the detection system. This signal drove the ultrasonic transducer (Panametrics, V314-SU) after being amplified to $45 \mathrm{~V}$ amplitude by the power amplifier and the transformer.

The operating bandwidth of the ultrasonic transducer was centered at $1 \mathrm{MHz}$. The diameter of the active element of the ultrasonic transducer was $1.9 \mathrm{~cm}$. The focal length of the transducer in water was $2.54 \mathrm{~cm}$. The diameter of the ultrasound focal spot in water was $0.2 \mathrm{~cm}$. The peak pressure at the focal spot underwater was 4.5 bars, whereas the maximum allowable diagnostic peak pressure inside human tissue is 50 bars. ${ }^{48,49}$

After light passed through the aperture and reached the PMT, the optical signal was converted into electrical signal. The electrical signal was separated into DC and AC components by an interface circuit. The DC voltage was read by a digital voltmeter, and the AC voltage was amplified and then effectively filtered by a narrow bandpass filter. The filtered signal was collected and averaged over 256 sweeps by the digital oscilloscope (Tektronix, 2440), which was triggered by a reference signal from the function generator. Both the frequency filtering and signal averaging enhanced the signal-to-noise ratio. The averaged time-domain signal was then transferred to a Macintosh computer for data storage and processing. The peak-to-peak voltage of the signal represented the light signal modulated by the ultrasound wave.

Once the time-domain signal in the oscilloscope was stored in the computer, the cuvette was moved to the next $(\mathrm{x}, \mathrm{z})$ position, and the above steps were repeated. When the signals of all 
the scanned positions were obtained, a one- or two-dimensional image of the turbid medium containing a buried object was constructed.

\section{Results}

\section{Ultrasound-Modulated Optical Signal in a Tissue Phantom}

To illustrate the effect of ultrasound modulation of light, we have selected a typical timedomain AC signal (Fig. 5a). The signal, consisting of 1,024 data points with a sampling interval of $0.04 \mu \mathrm{s}$, was transferred from the digital oscilloscope to the computer. The time-domain signal was converted to frequency-domain signal using FFT processing with one zero-padding ${ }^{50}$ and Hamming apodization ${ }^{51}$ (Fig. 5b). The zero-padding was used to increase the frequency resolution of the spectrum. The apodization was used to minimize the heights of the side lobes of the spectrum.

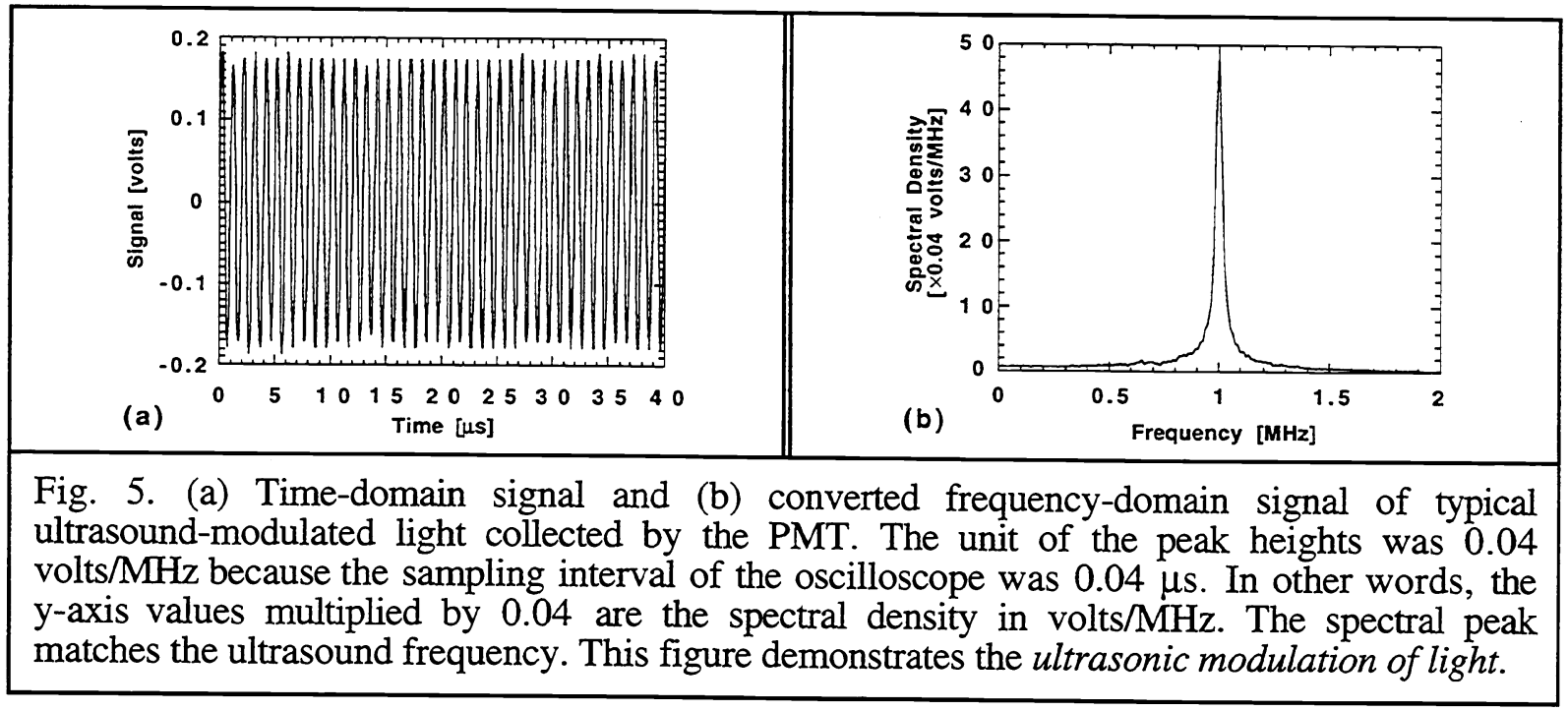

\section{Ultrasound-Modulated Optical Images of a Tissue Phantom}

A two-dimensional image of the above phantom was observed using the ultrasoundmodulated light signal (Fig. 6). The translation stage was scanned at $1 \mathrm{~mm}$ per step in both the $\mathrm{x}$ and $z$ directions. When the object was away from the ultrasound focus, the signal was strong. When the object was moved toward the ultrasonic focus, the signal dropped quickly.

The normalized one-dimensional images of the cube across $z=0$ using both the DC signals and the AC signals are shown in Fig. 7. The DC signals did not indicate the existence of a buried object. The AC signals showed a steep dip when the spheres were at the ultrasound focal spot. The imaging resolution (edge spread function) was $\sim 1 \mathrm{~mm}$, which was close to one half of the 2-mm focal-spot size of the ultrasound. 

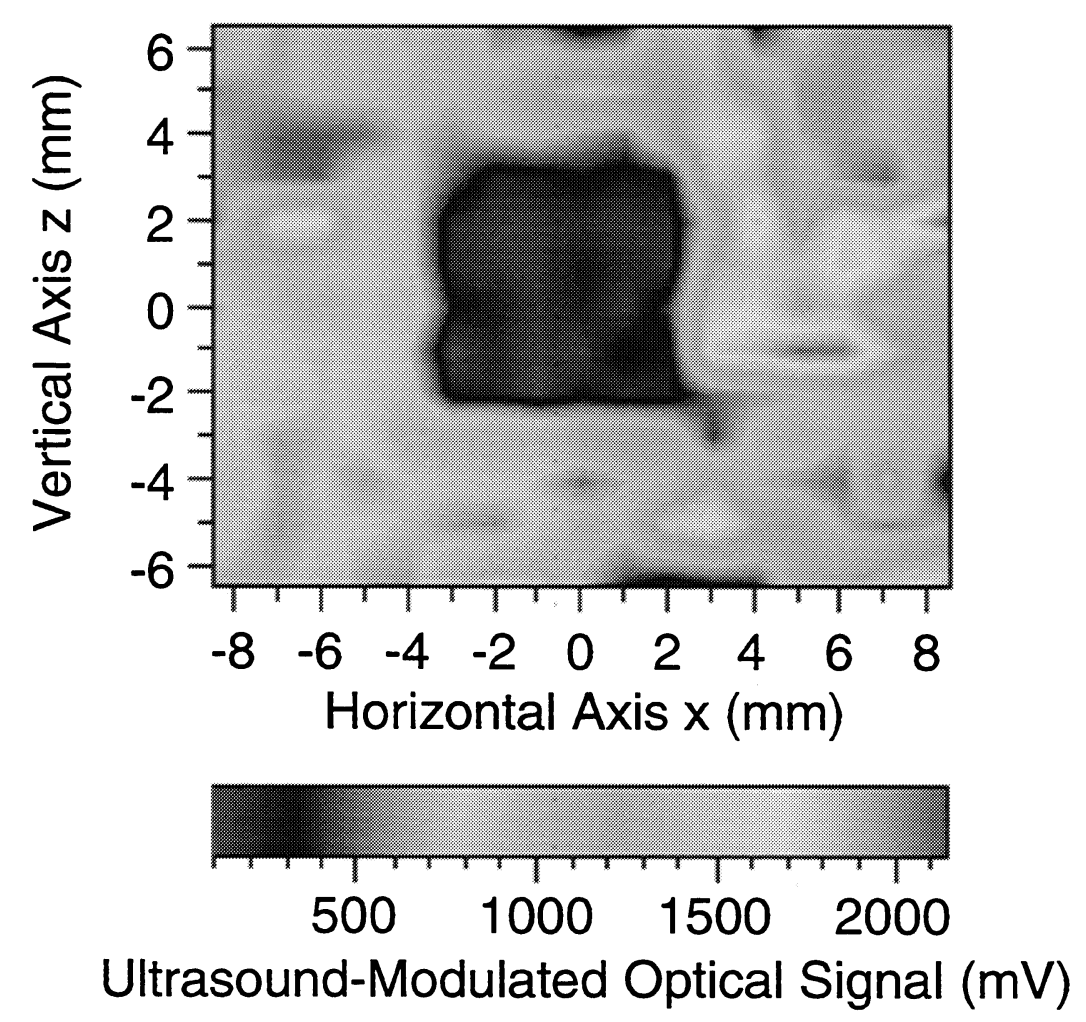

Fig. 6. Ultrasound-modulated optical tomography of a gel phantom.

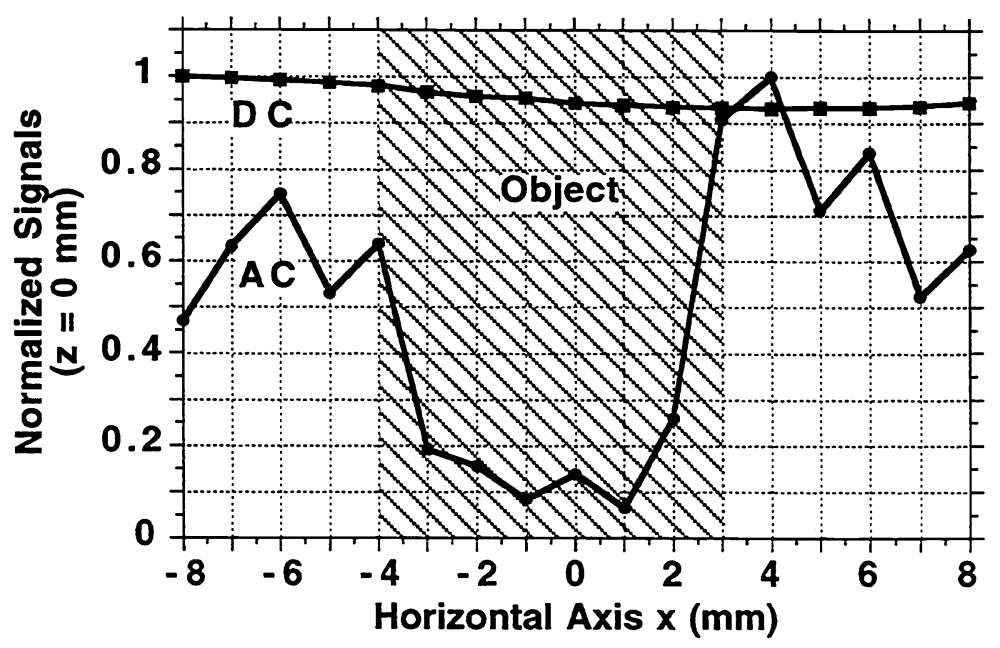

Fig. 7. Comparison of images using the ultrasound-modulated (AC) and unmodulated (DC) optical signals. 


\section{Discussion and Conclusions}

Since our first observation of ultrasound-modulated optical images of tenuous liquid turbid media to prove the concept, ${ }^{1}$ we have imaged dense gel tissue phantoms that simulated optical properties of breast tissues. The gel phantoms allowed us to bury objects without using a wire frame, which may disturb the ultrasonic field. The imaging resolution was $\sim 1 \mathrm{~mm}$. More studies are needed for quantitative imaging of the optical and mechanical properties of biological tissues.

\section{Acknowledgment}

The project was sponsored in part by The University of Texas M.D. Anderson Cancer Center grant, The Whitaker Foundation grant, Department of Energy grant, and National Institutes of Health grant.

\section{References}

1. Wang LH, Jacques SL, Zhao, XM. Continuous-wave ultrasonic modulation of scattered laser light to image objects in turbid media. Opt. Lett. 1995; 20:629-631.

2. Jackson VP. The role of US in breast imaging. Radiology 1990; 177:305-311.

3. Jackson VP, Hendrick RE, Feig, SA, Kopans DB. Imaging of the radiographically dense breast. Radiology 1993; 188:297-301.

4. Fornage BD, Lorigan JG, Andry E. Fibroadenoma of the breast: sonographic appearance. Radiology 1989; 172:671-675.

5. Fornage BD. Ultrasound of the breast. Ultrasound Quarterly 1993; 11:1-39.

6. Bassett LW, Kimme-Smith C. Breast sonography. Am. J. Roentgenol. 1991; 156:449-455.

7. Giger ML, Vyborny CJ, Schmidt RA. Computerized characterization of mammographic masses: analysis of speculation. Cancer Lett. 1994; 77:201-211.

8. Su B, Kappler F, Szwergold BS, Brown TR. Identification of a putative tumor marker in breast and colon cancer. Cancer Res. 1993; 53:1751-1754.

9. Zhou XH, Gordon R. Detection of early breast cancer: an overview and future prospects. Critical Rev. in Biomed. Eng. 1989; 17:203-255.

10. O'Leary MA, Boas DA, Chance B, Yodh AG. Experimental images of heterogeneous turbid media by frequency-domain diffusing-photon tomography. Opt. Lett. 1995; 20:426-428.

11. Yodh AG, Kaplan PD, Pine DJ. Pulsed diffusing-wave spectroscopy: high resolution through nonlinear optical gating. Phys. Rev B 1990; 42:4744-4747.

12. Hebden JC. Time-resolved imaging of opaque and transparent spheres embedded in a highly scattering medium. Appl. Opt. 1993; 32:3837-3841.

13. Hebden JC, Delpy DT. Enhanced time-resolved imaging with a diffusion model of photon transport. Opt. Lett. 1994; 19:311-313.

14. Chance B, Kang K, He L, Weng J, Sevick E. Highly sensitive object location in tissue models with linear in-phase and anti-phase multielement optical arrays in one and 2 dimensions. Proc. Nat. Acad. Sci. U.S.A. 1993; 90:3423-3427.

15. Feng L, Yoo KM, Alfano RR. Ultrafast laser-pulse transmission and imaging through biological tissues. Appl. Opt. 1993; 32:554-558.

16. Graber HL, Chang J, Lubowsky J, Aronson R, Barbour RL. Near infrared absorption imaging of dense scattering media by steady-state diffusion tomography. Proc. Soc. PhotoOpt. Instrum. Eng. 1993; 1888:372-386.

17. Hee MR, Izatt JA, Swanson EA, Fujimoto JG. Femtosecond transillumination tomography in thick tissues. Opt. Lett. 1993; 18:1107-1109.

18. Hee MR, Izatt JA, Jacobson JM, Fujimoto JG, Swanson EA. Femtosecond transillumination optical coherence tomography. Opt. Lett. 1993; 18:950-952. 
19. Knuttel A, Schmitt JM, Barnes R, Knutson JR. Acoustooptic scanning and interfering photon density waves for precise localization of an absorbing (or fluorescent) body in a turbid medium. Rev. Sci. Instrum. 1993; 64:638-644.

20. Haselgrove JC, Wang NG, Chance B. Investigation of the nonlinear aspects of imaging through a highly scattering medium. Med. Phys. 1992; 19:17-23.

21. Leith E, Chen C, Chen H, Chen Y, Dilworth D, Lopez J, Rudd J, Sun PC, Valdmanis J, Vossler G. Imaging through scattering media with holography. J. Opt. Soc. Am A 1992; 9:1148-1153.

22. Yoo KM, Xing QR, Alfano RR. Imaging objects hidden in highly scattering media using femtosecond second-harmonic-generation cross-correlation time gating. Opt. Lett. 1991; 16(13):1019-1021.

23. Yoo KM, Das BB, Alfano RR. Imaging of a translucent object hidden in a highly scattering medium from the early portion of the diffuse component of a transmitted ultrafast laser pulse. Opt. Lett. 1992; 17:958-960.

24. Duncan MD, Mahon R, Tankersley LL, Reintjes J. Time-gated imaging through scattering media using stimulated Raman amplification. Opt. Lett. 1991; 16:1868-1870.

25. Patterson MS, Moulton JD, Wilson BC, Berndt KW, Lakowicz JR. Frequency domain reflectance for the determination of the scattering and absorption properties of tissue. Appl. Opt. 1991; 30:4474-4476.

26. Andersson-Engels A, Berg R, Svanberg S, Jarlman O. Time-resolved transillumination for medical diagnostics. Opt. Lett. 1990; 15:1179-1181.

27. Jacques SL. Time resolved propagation of ultrashort laser pulses within turbid tissues. Appl. Opt. 1989; 28 (12):2223-2229.

28. Jacques SL. Time-resolved reflectance spectroscopy in turbid tissues. IEEE Trans. Biomed. Eng. 1989; 36 (12):1155-1161.

29. Jacques SL, Wang LH, Hielscher AH. Time-resolved photon propagation in tissues. In: A. J. Welch, M. J. C. van Gemert (ed.), Optical Thermal Response of Laser Irradiated Tissue, in press; Plenum Press, 1995.

30. Boas DA, O'Leary MA, Chance B, Yodh AG. Scattering and wavelength transduction of diffuse photon density waves. Phys. Rev. E 1993; 47:R2999-R3002.

31. Tromberg BJ, Svaasand LO, Tsay TT, Haskell RC. Properties of photon density waves in multiple-scattering media. Appl. Opt. 1993; 32:607-616.

32. Svaasand LO, Tromberg BJ, Haskell RC, Tsay TT, Berns MW. Tissue characterization and imaging using photon density waves. Optical Engineering 1993; 32:258-266.

33. Fishkin JB, Gratton E. Propagation of photon density waves in strongly scattering media containing an absorbing "semi-infinite" plate bounded by a straight edge. J. Opt. Soc. Am. A 1992; 10:127-134.

34. O'Leary MA, Boas DA, Chance B, Yodh AG. Refraction of diffuse photon density waves. Phys. Rev. Lett. 1992; 69:2658-2661.

35. Cheong WF, Prahl SA, Welch AJ. A review of the optical properties of biological tissues. IEEE J. Quantum Electronics 1990; 26:2166-2185.

36. Peters VG, Wyman DR, Patterson MS, Frank GL. Optical properties of normal and diseased human breast tissues in the visible and near infrared. Phys. Med. Biol. 1990; 35:1317-1334.

37. Wang LH, Jacques SL. Application of probability of $n$ scatterings of light passing through an idealized tissue slab in breast imaging. Proc. of Advances in Optical Imaging and Photon Migration, R. R. Alfano, ed., 1994; 181-186.

38. Wang LH, Stephens DV, Hielscher AH, Tittel FK, Jacques SL. Imaging of turbid media with a spatial filter," Proc. of Advances in Optical Imaging and Photon Migration, R. R. Alfano, ed., 1994; 288-290.

39. Moon JA, Mahon R, Duncan MD, Reintjes J. Resolution limits for imaging through turbid media with diffuse light. Opt. Lett. 1993; 18:1591-1593. 
40. Hall DJ, Hebden JC, Delpy DT. Time-resolved imaging of a solid breast phantom. Proc. Soc. Photo-Opt. Instrum. Eng. 1995; 2389:182-189.

41. Chance B, Kang K, He L, Weng J, Sevick E. Highly sensitive object location in tissue models with linear in-phase and anti-phase multielement optical arrays in one and 2 dimensions. Proc. Nat. Acad. Sci. U.S.A. 1993; 90:3423-3427.

42. Marks FA, Tomlinson HW, Brooksby GW. A comprehensive approach to breast cancer detection using light: photon localization by ultrasound modulation and tissue characterization by spectral discrimination. Proc. Soc. Photo-Opt. Instrum. Eng. 1993; 1888:500-510.

43. Ophir J, Cespedes I, Ponnekanti H, Yazdi Y, Li X. Elastography: a quantitative method for imaging the elasticity of biological tissues. Ultrason. Imaging 1991; 13:111-134.

44. Parker KJ, Huang SR, Musulin RA, Lerner RM. Tissue response to mechanical vibrations for "sonoelasticity imaging." Ultrasound Med. Biol. 1990; 16(3):241-246.

45. Duck FA. Physical properties of tissue: a comprehensive reference book. London, New York, Academic Press. 1990.

46. Korpel A. Acousto-optics. New York, Marcel Dekker, Inc. 1988.

47. Sapriel J. Acousto-optics. New York, John Wiley. 1979.

48. NCRP Report No. 74. Biological effects of ultrasound: mechanisms and clinical implications. National Council on Radiation Protection and Measurements. Bethesda, MD. 1983.

49. Whittingham TA. The safety of ultrasound. Imaging 1994, 6:33-51.

50. Press WH, Flannery BP, Teukolsky SA, and Vetterling WT. Numerical recipes in C. 2nd Ed., Cambridge Univ. Press, 1992.

51. Harris FJ. On the use of windows for harmonic analysis with the discrete Fourier transform. Proc. IEEE 1978; 66:51-83. 